\title{
Dependence of the Josephson Coupling of Unconventional Superconductors on the Properties of the Tunneling Barrier
}

M. Ledvij and R. A. Klemm
Materials Science Division
Argonne National Laboratory

Argonne, IL 60439

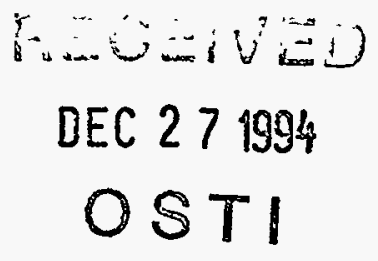

The submithed manuscript has been authored by a Wontractor of the U.S. Government Under contract No retains a nonexclusive, royatty-tree license to publish o

reproduce the published form $\alpha$ this contribution, or allow

ahers to do so, for U.S. Government purposes.

May 1994

$/ \mathrm{sm}$

This work is supported by the Division of Materials Sciences, Office of Basic Energy Sciences of DOE, under contract No. W-31-109-ENG-38. 


\section{DISCLAIMER}

Portions of this document may be illegible in electronic image products. Images are produced from the best available original document. 
May 30, 1994

\title{
Dependence of the Josephson coupling of unconventional superconductors on the properties of the tunneling barrier
}

\author{
M. Ledvij and R. A. Klemm \\ Materials Science Division, Argonne National Laboratory, Argonne, IL 60439
}

\begin{abstract}
The Josephson coupling between a conventional and an unconventional superconductor is investigated as a function of the properties of the tunneling barrier. A simple model is adopted for the tunneling probability and it is shown that its variation dramatically affects the $I_{c} R_{n}$ product of an s-d, as. opposed to an s-s junction. Based on these conclusions, experiments are proposed to probe the symmetry of the order parameter in high temperature superconductors.
\end{abstract}

\section{DISCLAIMER}

This report was prepared as an account of work sponsored by an agency of the United States Government. Neither the United States Government nor any agency thereof, nor any of their employees, makes any warranty, express or implied, or assumes any legal liability or responsibility for the accuracy, completeness, or usefulness of any information, apparatus, product, or process disclosed, or represents that its use would not infringe privately owned rights. Reference herein to any specific commercial product, process, or service by trade name, trademark, manufacturer, or otherwise does not necessarily constitute or imply its endorsement, recommendation, or favoring by the United States Government or any agency thereof. The views and opinions of authors expressed herein do not necessarily state or reflect those of the United States Government or any agency thereof. 
One of the highly contested subjects in the condensed matter physics is the symmetry of the order parameter in high temperature superconductors, which is closely related to the question of the pairing mechanism. Recent experiments on photoemission in some of these superconductors suggest that the order parameter is very anisotropic [1], possibly with regions in $k$-space where it is exactly zero, although other interpretations have also been suggested [2]. The measurements of the magnetic penetration depth $\lambda$ have given power law temperature $(T)$ dependences that are in disagreement with the standard BCS result. In particular, the linear $T$-dependence of $\lambda(T)$ observed in very clean $\mathrm{YBa}_{2} \mathrm{Cu}_{3} \mathrm{O}_{7-\delta}(\mathrm{YBCO})$ single crystals [3], has been interpreted in terms of an order parameter with line nodes on the Fermi surface. Both the photoemission and the penetration depth measurements have been widely cited as giving support to the contention that the order parameter in high temperature superconductors has $d$-wave symmetry. However, these two families of experiments can only suggest whether there are low lying excitations in the superconducting state, which is possible both in $d$-wave and anisotropic $s$-wave superconductors, and also in gapless, proximity-coupled SN multilayer models, [4], without addressing the question of the sign of the order parameter. This problem has recently been dealt with by several experimental groups [5-10], who considered Josephson coupling of high temperature superconductors in different geometries. All these experiments are based on a unique idea $[11,12]$ that the critical current of a junction must decisively depend on the orientation of the crystalographic axes if at least one of the superconductors has $d$-wave symmetry. This feature brings about a qualitative difference from the case of Josephson coupling between two $s$-wave superconductors. Nevertheless, the experiments have so far provided contradicting conclusions, some of them suggesting $s$-wave $[6,8]$ and some $d$-wave $[5,9,10]$ symmetry (the experiments by the Urbana and ETH groups [5,9] have recently been given an alternative interpretation [13]).

In this Letter we address the problem from a different side. We show how the properties of the tunneling barrier can significantly alter the Josephson coupling if one of the superconductors involved has a gap function (such as that with $d$-wave symmetry) which averages to zero, thereby providing another possibility to test the symmetry of the order parameter. 
From the quantum mechanical point of view a tunneling junction is usually described by the Hamiltonian which transfers electrons from one metal to the other:

$$
\mathcal{H}_{T}=\sum_{\mathbf{p}, \mathbf{q}, \sigma}\left(T_{\mathbf{p}, \mathbf{q}} c_{\mathbf{p} \sigma}^{+} d_{\mathbf{q} \sigma}+T_{\mathbf{p}, \mathbf{q}}^{*} d_{\mathbf{q} \sigma}^{+} c_{\mathbf{p} \sigma}\right)
$$

where $c_{\mathbf{p} \sigma}^{+}\left(c_{\mathbf{p} \sigma}\right)$ creates (destroys) an electron with momentum $\mathrm{p}$ and spin $\sigma$ in metal 1 , while $d_{\mathbf{q} \sigma}^{+}\left(d_{\mathbf{q} \sigma}\right)$ creates (destroys) an electron with momentum $\mathbf{q}$ and spin $\sigma$ in metal 2 . The quantity $T_{\mathbf{p}, \mathbf{q}}$ is a matrix element connected to the transition probability for an electron from a $\mathbf{p}$-state on side 1 to a $\mathbf{q}$-state on side 2 . We assume units in which $\hbar=k_{B}=c=1$.

The current through the junction is evaluated as $I=-e \dot{N}_{i}=-i e\left[\mathcal{H}_{T}, N_{i}\right]$, where $N_{i}$ is the operator of the number of particles on the $i^{\text {th }}$ side. To the lowest order in the perturbation theory, one gets for the current in the normal state:

$$
I_{n}=4 \pi e \sum_{\mathbf{p q}}\left|T_{\mathbf{p}, \mathbf{q}}\right|^{2}\left[f\left(\xi_{1 \mathbf{p}}-e V\right)-f\left(\xi_{2 \mathbf{q}}\right)\right] \delta\left(\xi_{1 \mathbf{p}}-\xi_{2 \mathbf{q}}\right),
$$

where $\xi_{1 \mathrm{p}}$ and $\xi_{2 q}$ are the energy dispersions in the two metals relative to the Fermi level, and $f$ is the Fermi function [14]. The Josephson critical current is obtained as

$$
I_{c}=2 e \sum_{\mathbf{p q}}\left|T_{\mathrm{p}, \mathrm{q}}\right|^{2} \frac{\Delta_{1}(\mathrm{p}) \Delta_{2}(\mathrm{q})}{\varepsilon_{1 \mathrm{p}} \varepsilon_{2 \mathrm{q}}} \frac{\varepsilon_{1 \mathrm{p}} \tanh \left[\varepsilon_{2 \mathrm{q}} /(2 T)\right]-\varepsilon_{2 \mathrm{q}} \tanh \left[\varepsilon_{1 \mathrm{p}} /(2 T)\right]}{\varepsilon_{1 \mathrm{p}}^{2}-\varepsilon_{2 \mathrm{q}}^{2}},
$$

where $\Delta_{1}(p)$ and $\Delta_{2}(q)$ are the gap functions in the two metals, with $\varepsilon_{1 \mathrm{p}}=\sqrt{\xi_{1 \mathrm{p}}^{2}+\left|\Delta_{1}(\mathrm{p})\right|^{2}}$ and similarly for the other metal. We allow the two metals to have different Fermi surfaces as well as gap functions.

Hereafter we will restrict ourselves to $T=0$. Under the assumption that both superconductors are of the BCS type with spherical Fermi surfaces and constant gaps $\left[\Delta_{1}(p)=\Delta_{1}\right.$, $\Delta_{2}(q)=\Delta_{2}$ ], Ambegaokar and Baratoff derived from (2) and (3) the following expression for the product of the critical current and the normal state resistance of the junction $[15,14]$ :

$$
e I_{c} R_{n}=\frac{2 \Delta_{1} \Delta_{2}}{\Delta_{1}+\Delta_{2}} K\left(\frac{\left|\Delta_{1}-\Delta_{2}\right|}{\Delta_{1}+\Delta_{2}}\right)
$$

with $K$ being the complete elliptic integral of the first kind. The most important feature of this formula is that the measurable quantity $I_{c} R_{n}$ depends on the gap values of the two 
metals and is independent of the properties of the barrier. We shall show that this result also holds for Josephson tunneling between $s$-wave superconductors with arbitrary Fermi surfaces and tunneling directions. However, the situation changes dramatically when the order parameter in at least one of the two superconductors has $d$-wave symmetry.

In what follows we assume that an isolated metal 1 has a free-electron-like dispersion $\xi_{1 \mathrm{p}}=p^{2} /\left(2 m_{1}\right)-E_{F 1}$ with the s-wave (isotropic) gap $\Delta_{1}(\mathrm{p}) \equiv \Delta_{1}$. An insulated metal 2 has either an uniaxially ellipsoidal Fermi surface with a dispersion $\xi_{2 q}=\left(q_{x}^{2}+q_{y}^{2}\right) /\left(2 m_{2}\right)+$ $q_{z}^{2} /\left(2 M_{2}\right)-E_{F 2}$, or an open Fermi surface appropriate for a layered conductor, $\xi_{2 q}=\left(q_{x}^{2}+\right.$ $\left.q_{y}^{2}\right) /\left(2 m_{2}\right)+J\left(1-\cos q_{z} s\right)-E_{F 2}$, where $J \ll E_{F 2}$, and a gap function which depends only on the $x$ and $y$ components of the wave-vector, $\Delta_{2}(q) \equiv \Delta_{2}\left[q \equiv\left(q_{x}^{2}+q_{y}^{2}\right)^{1 / 2}, \varphi\right]$. Bringing these metals into contact changes their Fermi energies so that $E_{F 1}=E_{F 2}$.

The properties of the tunneling barrier are buried in the matrix elements $\left|T_{\mathbf{p}, \mathbf{q}}\right|^{2}$ giving the transition probability for an electron tunneling from the state $\mathrm{p}$ on one side to the state $q$ on the other side of the barrier. To get an insight into the importance of the properties of the barrier for the Josephson tunneling, we assume that the transition probability has the following simple form [16]:

$$
\left|T_{\mathrm{p}, \mathrm{q}}\right|^{2}=\exp \left[-\frac{p^{2}-(\mathrm{p} \cdot \hat{n})^{2}}{(\mathrm{p} \cdot \hat{n})^{2} \theta_{0}^{2}}\right] \exp \left[-\frac{q^{2}-(\mathrm{q} \cdot \hat{n})^{2}}{(\mathrm{q} \cdot \hat{n})^{2} \theta_{0}^{2}}\right]
$$

The unit vector $\hat{n}$ is perpendicular to the plane of the junction. The two exponents restrict the wave-vectors of the tunneling eletrons to those that are nearly perpendicular to the plane of the junction. In that respect, the parameter $\theta_{0}$ roughly corresponds to the tangent of the maximum angle between the quasimomentum of the electrons which are able to tunnel with a nonnegligible probability, and the normal to the junction. For $\theta_{0} \ll 1$, only those electrons that are perpendicular to the junction will be able to tunnel, whereas for $\theta_{0} \gg 1$ there will be practically no restrictions in that the probability of the electrons to tunnel will be almost the same regardless of the angle of their quasimomenta relative to the plane of the junction.

We will assume for the moment that the gap in the second metal has $d$-wave symmetry: 
$\Delta_{2}(q) \equiv \Delta_{2} \cos 2 \varphi$, where $\varphi$ is the azimuthal angle in the $q_{x}-q_{y}$ plane, and $\Delta_{2}$ is the maximum value of the gap. Let us consider two different geometries. We assume there is a single domain of this gap function, which is locked onto the crystal lattice.

First, let the plane of the junction be parallel to the $a-b(x-y)$ plane of the second metal: $\hat{n}=\hat{z}$ (this is the geometry probed recently in the experiment on $\mathrm{YBCO}-\mathrm{Pb}$ junctions [8]). The form of $\left|T_{\mathbf{p}, \mathbf{q}}\right|^{2}, \mathrm{Eq}$. (5), then implies that the tunneling electrons do not have a preferrential quasimomentum orientation in the $x-y$ plane [the exponents in (5) are $\varphi-$ independent]. It is easy to see from Eq. (3) that this produces zero critical current since there is exact cancellation of the positive and negative regions in q-space. This conclusion does not depend on the value of $\theta_{0}$.

Next, let the plane of the junction contain the $z$-axis, with $\beta$ being the angle between the normal to the plane of the junction and the $x$ axis: $n_{z}=0, \cos \beta=n_{x}$. Then, using Eqs. (2), (3) and (5), after some algebra, one obtains for the critical current the following expression:

$$
e I_{c} R_{n}=\frac{2 \int_{0}^{2 \pi} d \varphi \frac{\Delta_{1} \Delta_{2} \cos 2 \varphi}{\Delta_{1}+\Delta_{2}|\cos 2 \varphi|} K\left(\frac{\left|\Delta_{1}-\Delta_{2}\right| \cos 2 \varphi \mid}{\Delta_{1}+\Delta_{2}|\cos 2 \varphi|}\right) g(\varphi-\beta)}{\int_{0}^{2 \pi} d \varphi g(\varphi)} .
$$

Here, both $\Delta_{1}$ and $\Delta_{2}$ are taken to be positive, with

$$
g(\varphi)=\exp \left(-\frac{\tan ^{2} \varphi}{\theta_{0}^{2}}\right) \int_{0}^{1} \frac{d \rho}{\sqrt{\rho}} \exp \left(-\frac{M_{2} / m_{2}}{\theta_{0}^{2} \cos ^{2} \varphi} \frac{\rho}{1-\rho}\right)
$$

for the uniaxially ellipsoidal dispersion, and

$$
g(\varphi)=|\cos \varphi| \operatorname{erf}\left(\frac{\pi / s q_{F}}{|\cos \varphi| \theta_{0}}\right) \exp \left(-\frac{\tan ^{2} \varphi}{\theta_{0}^{2}}\right)
$$

for the open Fermi surface ( $q_{F}$ is the Fermi wave-vector). First thing to notice is that the Ambegaokar-Baratoff result, Eq. (4), is recovered if $\cos 2 \varphi$ in (6) is formally replaced with unity, which would correspond to both metals having (isotropic) s-wave gaps. That holds true even if all three effective masses are different. The most remarkable feature of $(6)$ is the way in which the angular dependence of the gap in the $d$-wave metal is coupled to 
the angular dependence of the tunneling probability contained in the function $g(\varphi)$. This coupling comes from the fact that the geometry of the junction now breaks the $x-y$ symmetry in the $d$-wave superconductor, producing nonvanishing critical current. To discern the role of the barrier parameter $\theta_{0}$, the product $I_{c} R_{n}$ is shown in Fig. 1 as a function of the angle $\beta$ for several values of $\theta_{0}$ for the case of the open Fermi surface (the conclusions for the uniaxially ellipsoidal dispersion are the same). All the curves are qualitatively similar they are antisymmetric with respect to $\beta=45^{\circ}$ - the angle when the node direction of the $\mathrm{d}$-wave gap is perpendicular to the junction. However, there are substantial quantitative differences for different $\theta_{0}$. The $I_{c} R_{n}$ product is the largest in the limit $\theta_{0} \rightarrow 0$. In that case the function $g(\varphi)$, Eqs. (7) and (8), behaves like a delta function so that the product $I_{c} R_{n}$ becomes:

$$
e I_{c} R_{n}=\frac{2 \Delta_{1} \Delta_{2} \cos 2 \beta}{\Delta_{1}+\Delta_{2}|\cos 2 \beta|} K\left(\frac{\left|\Delta_{1}-\Delta_{2}\right| \cos 2 \beta||}{\Delta_{1}+\Delta_{2}|\cos 2 \beta|}\right) .
$$

The physical meaning of this formula is the following: when $\theta_{0}$ is small, only the Cooper pairs with quasimomenta almost perpendicular to the junction take part in tunneling, and the gap value for that particular direction is the only one relevant; that is why the formula looks very much like the Ambegaokar-Baratoff result, Eq. (4). Note that it differs somewhat from the widely adopted formula $I_{c} \propto \cos 2 \beta$ [12]. The $I_{c} R_{n}$ product drops significantly when $\theta_{0}$ increases (see also Fig. 2). The reason for this is that Cooper pairs with a range of the quasimomentum angles contribute now to the Josephson current, so that there is a substantial cancellation of the positive and negative regions of $\Delta(q)$ stemming from its $d$-wave nature. In the limit $\theta_{0} \rightarrow \infty$, the function $g(\varphi)$ tends to a constant, causing the Josephson current to vanish, since in that case the junction does not act anymore as an $x-y$ symmetry-breaker for the $\mathrm{d}$-wave superconductor.

As an interesting comparison, we give in Fig. 3 the $I_{c} R_{n}$ product for a Josephon junction characterized by the same tunneling probability (5), but with the $d$-wave superconductor being replaced with an extremely anisotropic $s$-wave superconductor having $\Delta_{2}(q) \equiv \Delta_{2}|\cos 2 \varphi|$. For small $0_{0}$ the critical current has some angular dependence due to 
the selectivity of the barrier with respect to the electron quasimomenta. With increasing $\theta_{0}$ the critical current gets "isotropized" without losing its magnitude, since the gap function now has the same sign everywhere in q-space and there is no cancellation.

How can we justify the use of the tunneling probability as given in Eq. (5)? The simplest thing to assume that the longer the "path" of the electrons through the barrier, the smaller the tunneling probability. Therefore, the electrons tunneling in the perpendicular direction should have the highest probability, which is what (5) implies. Another interpretation would involve possible scattering inside the barrier which tends to "isotropize" the tunneling probability, whereby effectively increasing the parameter $\theta_{0}$. In that respect, the effect of junction scattering on the s-d Josephson current would be very much like that of bulk scattering upon the transition temperature of a $d$-wave superconductor.

How can we use the main result of this paper (Fig. 1 as opposed to Fig. 3) to probe the symmetry of the order parameter in high temperature superconductors? Although the theoretical approach used here assumes the contact to be of SIS' type, the physical idea applies equally well to SNS' type junctions. What we propose to be done is to progressively introduce (nonmagnetic) impurities into the normal layer of an SNS' contact where one of the superconductors is conventional (believed to be $s$-wave), while the other one is an high $T_{c}$ superconductor. The depression of the critical current due to impurities should be much stronger if the high $T_{c}$ metal has $d$-wave symmetry, as opposed to both metals having $s$-wave order parameters. What exactly "much stronger" means, is best determined experimentally: together with the proposed series of SNS' Josephson junctions, one ought to make a series of SNS Josephson junctions with both superconductors being conventional, and with varying concentrations of impurities in the normal junction layers; the same metal and the same impurities should be used for the normal junction layers in both series.

A less reliable possibility is to use point contacts. If a tip made of a conventional superconductor is put in contact with a flat surface of a high temperature superconductor, the parameter $\theta_{0}$ in the tunneling probability (5) could be made larger or smaller depending on the distance as well as the opening angle of the tip: the larger the opening angle, the 
larger $\theta_{0}$, assuming that the tip touches the surface. However, this method is difficult, at best, to control.

The authors would like to acknowledge discussions with A. A. Abrikosov, M. R. Beasley, Jun Chen, R. C. Dynes, D. A. Gajewski, K. Gray, L. H. Greene, M. B. Maple, A. G. Sun, and J. Zasadzinski. This work has been supported by the US Department of Energy, Division of Basic Energy Sciences, through contract No. W-31-109-ENG-38. 


\section{REFERENCES}

[1] Z. -X. Shen et al., Phys. Rev. Lett. 70, 1553 (1993).

[2] R. Fehrenbacher and M. Norman, preprint; S. H. Liu and R. A. Klemm, preprint.

[3] W. N. Hardy et al., Phys. Rev. Lett. 70, 3999 (1993).

[4] A. A. Abrikosov and R. A. Klemm, Physica C 191, 224 (1992); R. A. Klemm and S. H. Liu, Physica C191, 398 (1992) and preprint (1994).

[5] D. A. Wollman et al., Phys. Rev. Lett. 71, 2134 (1993).

[6] P. Chaudhari and S. Y. Lin, Phys. Rev. Lett. 72, 1084 (1994).

[7] H. Z. Durusoy, D. Lew, L. Lombardo, A. Kapitulnik, T. H. Geballe, and M. R. Beasley, preprint.

[8] A. G. Sun et al., Phys. Rev. Lett. 72, 2267 (1994).

[9] D. A. Brawner and H. R. Ott, preprint.

[10] C. C. Tsuei, J. R. Kirtley, C. C. Chi, L. S. Yu-Jahnes, A. Gupta, T. Shaw, J. Z. Sun, and M. B. Ketchen, preprint.

[11] V. B. Geshkenbein and A. I. Larkin, Pis'ma Zh. Eksp. Teor. Fiz. 43, 306 (1986) [JETP Lett. 43, 395 (1986)].

[12] M. Sigrist and T. M. Rice, J. Phys. Soc. Jap. 61, 4283 (1992).

[13] R. A. Klemm, submitted as a Comment to Phys. Rev. Lett.

[14] See, e.g., A. A. Abrikosov, Fundamentals of the Theory of Metals, North-Holland, Amsterdam, New York, 1988.

[15] V. Ambegaokar and A. Baratoff, Phys. Rev. Lett. 10, 486 (1963); 11, 104 (1963).

[16] The dimensional preexponential constants are omitted since they do not contribute to 
$I_{c} R_{n}$. 


\section{FIGURES}

FIG. 1. The reduced $I_{c} R_{n}$ product of the s-d junction as a function of the angle $\beta$ between the $x$ axis of the $d$-wave superconductor and the normal to the junction, for $\Delta_{1} / \Delta_{2}=0.1, \pi / s q_{F}=1$, and for three different values of the barrier parameter $\theta_{0}$.

FIG. 2. The $I_{c} R_{n}$ of the s-d junction for $\beta=0$, scaled with $I_{c} R_{n}$ for an s-s junction (Ambegaokar-Baratoff, both s-wave superconductors having isotropic BCS gaps) for the same choice of the transition temperatures of the two metals. The limiting value for $\theta_{0} \rightarrow 0$ is greater than 1 because $\Delta_{2} \approx 2.14 T_{c 2}$ for a d-wave metal, while $\Delta_{2} \approx 1.76 T_{c 2}$ for an s-wave metal.

FIG. 3. The reduced $I_{c} R_{n}$ product of an $s-s$ junction as a function of the angle $\beta$ between the $x$ axis of the anisotropic s-wave superconductor (see text) and the normal to the junction, for $\Delta_{1} / \Delta_{2}=0.1, \pi / s q_{F}=1$, and for three different values of the barrier parameter $\theta_{0}$. 


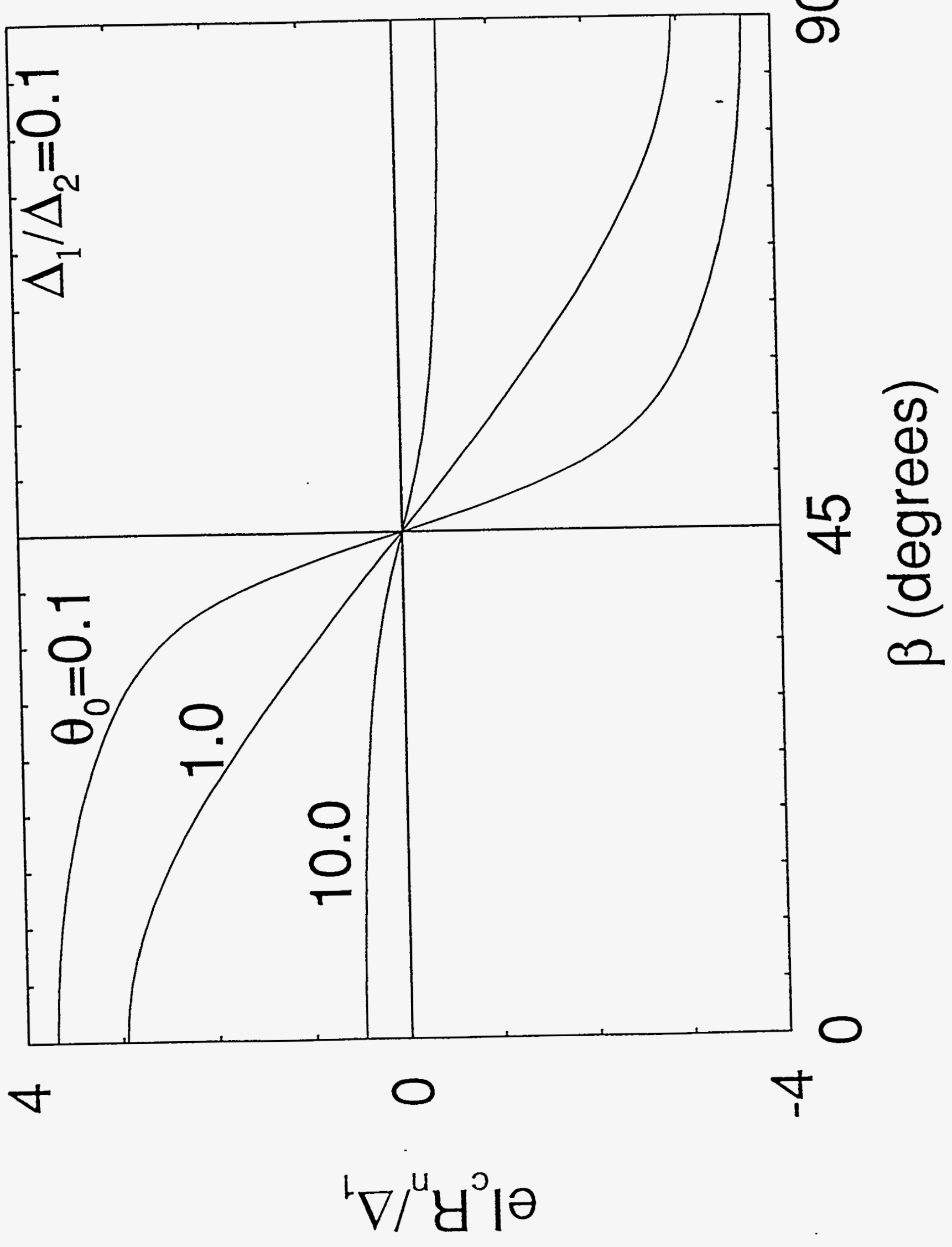




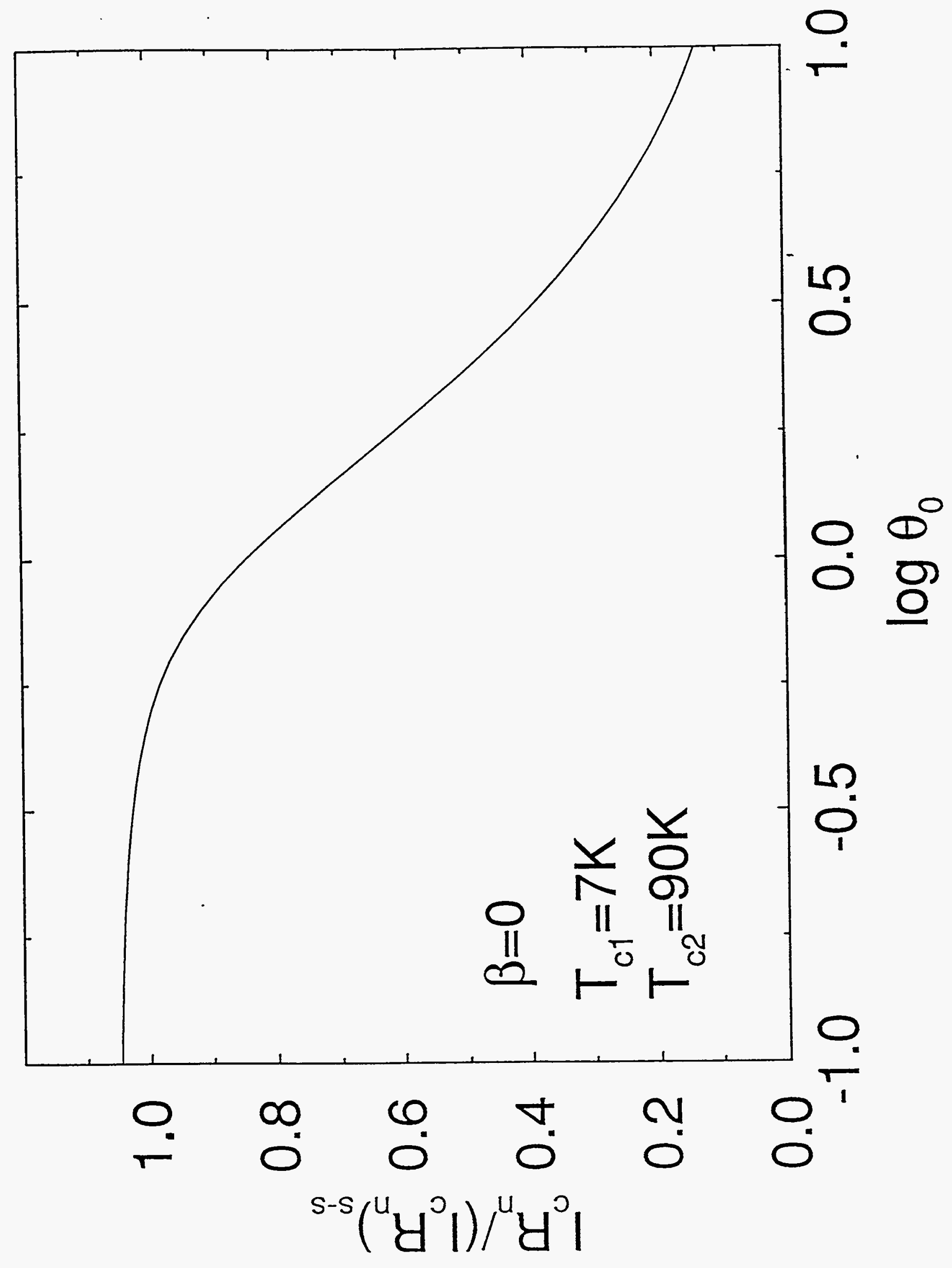




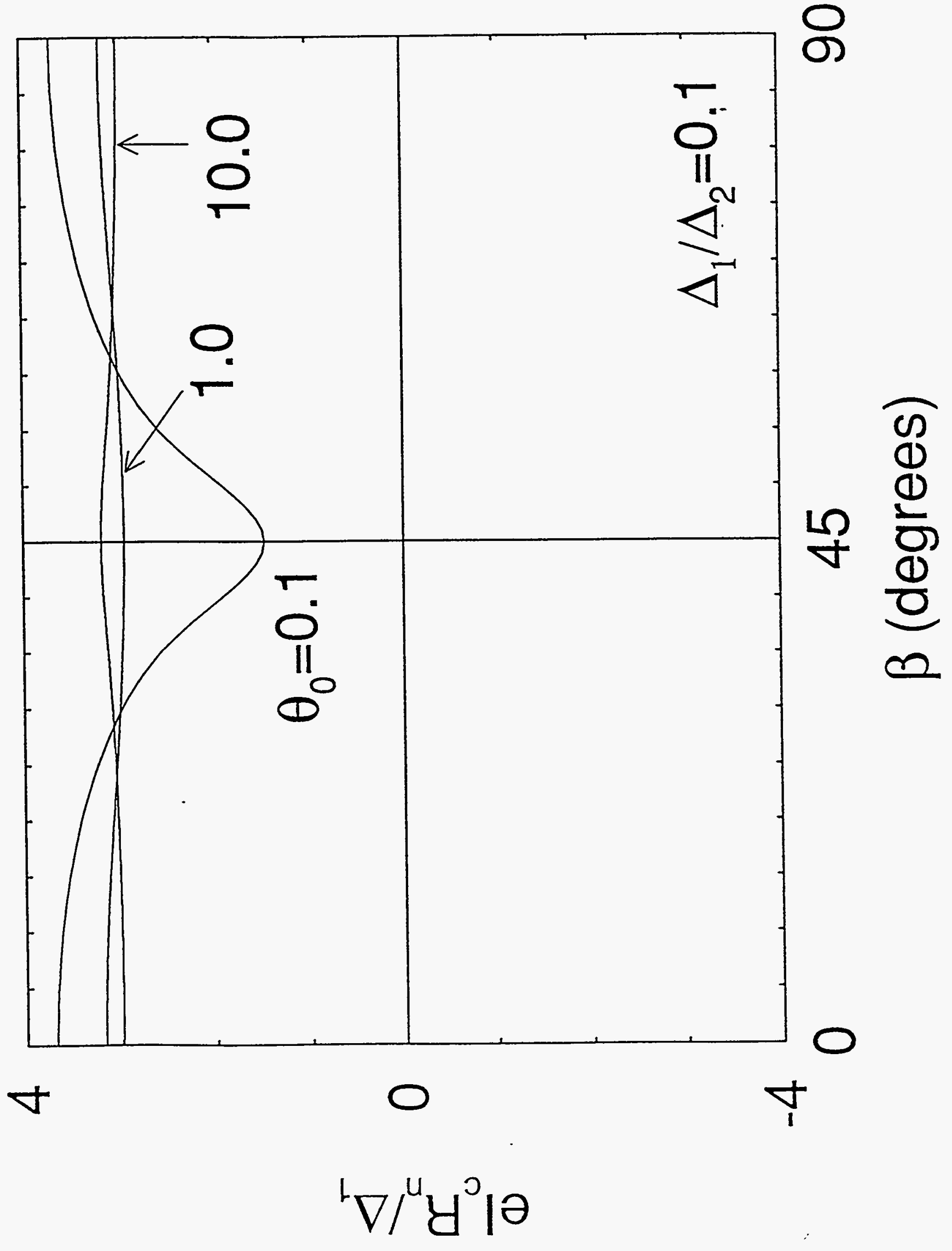

